\title{
Interfacial Electronic Structure in Graded Shell Nanocrystals Dictates their Performance for Optical Gain
}

\author{
Brenna R. Walsh ${ }^{\dagger}$, Jonathan I. Saari ${ }^{\dagger}$, Michael M. Krause ${ }^{\dagger}$, Timothy Mack ${ }^{\dagger}$ Robert Nick ${ }^{\star}$, Seth \\ Coe-Sullivan ${ }^{*}$ Patanjali Kambhampati ${ }^{*} *$ \\ ${ }^{\dagger}$ Chemistry, McGill, Montreal, H3A 0B8, Canada \\ ${ }^{\ddagger}$ QD Vision Inc., 29 Hartwell Ave., Lexington, MA, 02421, USA
}

Here further details of the transient absorption spectra are shown. In figure S1, a series of kinetic transients are shown for each of the four pumps (X1-X3 and $400 \mathrm{~nm}$ ). When pumping with $\mathrm{X} 1$, figure $\mathrm{S} 1 \mathrm{a}$, the change in optical density begins to grow in at the $\mathrm{B} 1$ peak, before the $\mathrm{B} 2$ peak, peak nomenclature taken from Klimov et. al ${ }^{1-2}$. However, by 500 fs, both the B1 and B2 peaks begin to evolve simultaneously, and show the largest change in optical density by $500 \mathrm{fs}$. When pumping with X2 (fig. S1 b), growth begins at early time (by $~ 100 \mathrm{fs}$ ) for both the B1 and B2 peaks, and growth continues for $\geq 1$ ps. The growth of the B2 peak is maximized at $1 \mathrm{ps,}$ while the B1 peak continues growth for 5 ps. Different than with X2 pumping, the B1 and B2 peaks obtained when pumping at $\mathrm{X} 3$ grow together and growth for both is maximized at $1 \mathrm{ps}$. Both peaks have begun to shrink by $50 \mathrm{ps}$. 

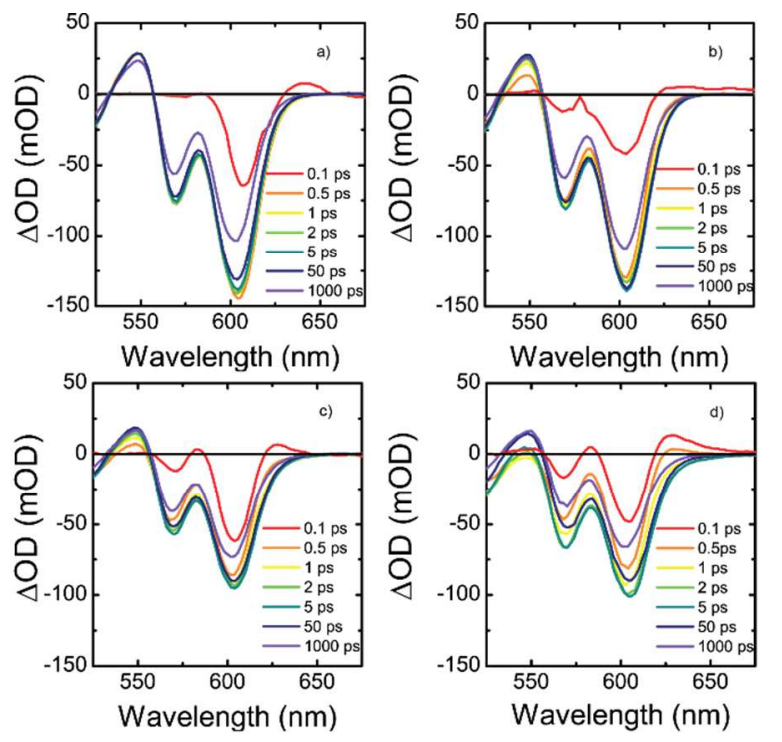

Figure S1. Shows transient absorption spectra when pumping X1-X3 as well as pumping with $400 \mathrm{~nm}$ in panels a.-d.. The sample is excited with power of $<\mathrm{N}>\sim 0.5$, so only a single excitation per dot is expected. Spectra show increasing pump/probe time delays from 100 fs to 1 ns.
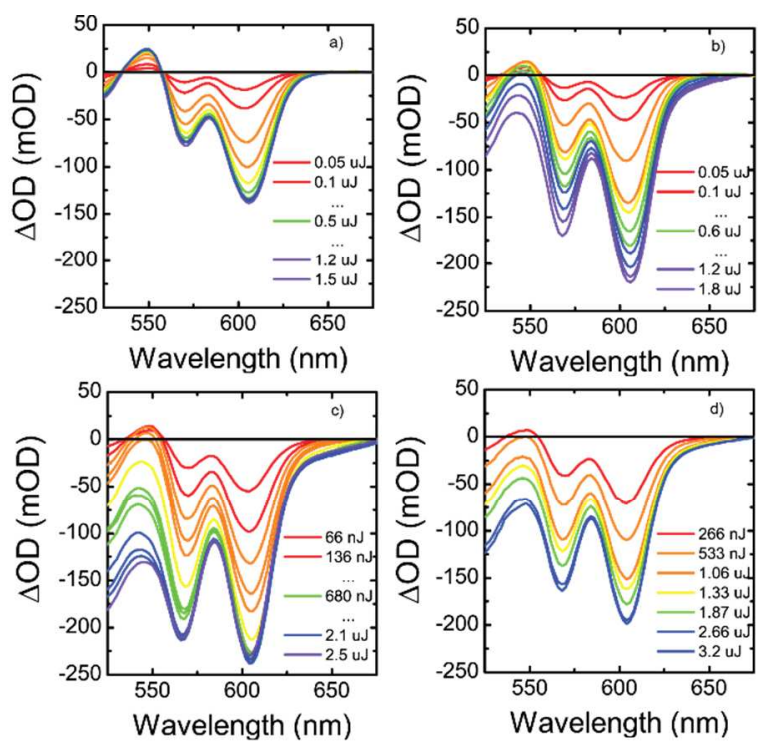
Figure S2. Transient absorption spectra at pump/probe time delay of 2 ps for pumps X1-X3 and $400 \mathrm{~nm}$, panels a.-d.. Transients show a variety of fluences for each pump.

In figure S2, the sample is pumped at the X1-X3 transitions, as well as with $400 \mathrm{~nm}$, panels ad. The pump/probe time delay is fixed, and a series of spectra are shown with increasing pump fluence.

When pumping the $\mathrm{X} 1$ transition, the B1 and B2 peaks grow in simultaneously and both peaks saturate at $1.2 \mu \mathrm{J}$. A distinct A2 signal is also observed, which is distinct from the bleach signals as the signal in the A2 region clearly shows absorption at all fluences. This is attributed to state filling of the first S type energy level, as the X1 transition will only access this first state. When pumping the $\mathrm{X} 2$ transition, similar growth is seen for the B1 and B2 transitions. However, though the X2 transition is expected to be S-like, we do not see this distinct absorbing feature in the A2 region. At low fluences, the A2 transition shows absorption, but as fluence is increased, a bleach is observed in this region. This bleaching in the region of the A2 feature is also observed when pumping at X3. This transition is expected to be P-like, therefore accessing higher lying states is expected for this transition. This is an alternate illustration of the hypothesis that the X2 transition is not purely S-like, and that it may have some P-like character, as initially proposed from the non-linear absorption spectra (Figure 3) and following analysis in the main text.

The following table shows the transient absorption and non-linear absorption values which were obtained at the band-edge when pumping with an X2 pump at a variety of different fluences, the first trial corresponding to spectra from the main text, figure $4 \mathrm{~b}$. Fluences were selected above and below the gain threshold $\left(\mathrm{N}_{\text {th }}\right)$ to compare this value to others seen for similar systems. The gain behavior observed when pumping resonant to the $\mathrm{X} 2$ transition was not as expected. Details of the nature of this anomaly are given in the main text, but it is important to 
highlight here that there $<\mathrm{N}>$ values were extracted by fitting the data to a Poisson distribution, which is not typically done for a doubly degenerate state.

Table S1. Average number of excitations per particle when pumping into the X2 state

\begin{tabular}{|c|c|c|c|}
\hline \multicolumn{4}{|c|}{ X2 Pump, Trail 1} \\
\hline Power (mJ) & $\Delta \mathrm{OD}$ & $-\Delta \mathrm{OD} / \mathrm{OD}_{0}$ & $<\mathrm{N}>$ \\
\hline 0.05 & -0.0234 & 0.1239 & 0.25 \\
\hline 0.1 & -0.0471 & 0.2494 & 0.52 \\
\hline 0.2 & -0.0907 & 0.4799 & 1.09 \\
\hline 0.3 & -0.1352 & 0.7153 & 1.93 \\
\hline 0.4 & -0.1450 & 0.7671 & 2.2 \\
\hline 0.5 & -0.1646 & 0.8710 & 2.96 \\
\hline 0.6 & -0.1795 & 0.9495 & 4.1 \\
\hline 0.7 & -0.1870 & 0.9895 & 5.94 \\
\hline 0.9 & -0.2023 & & \\
\hline 1.2 & -0.2119 & & \\
\hline 1.8 & -0.2182 & & \\
\hline
\end{tabular}

Table S2 shows the values for Stokes shifts and binding energies received for the three CdSe NCs studied previously by $\mathrm{Sewall}^{3}$ and for the $\mathrm{CdSe} / \mathrm{Cd}, \mathrm{Zn}, \mathrm{S}$ nanocrystal sample. It is clearly visible that none of the values for $\mathrm{CdSe} / \mathrm{Cd}, \mathrm{Zn}, \mathrm{S}$ follow the trend seen for $\mathrm{CdSe}$.

Table S2. Comparison of $\mathrm{CdSe}$ and $\mathrm{CdSe} / \mathrm{Cd}, \mathrm{Zn}, \mathrm{S}$ Stokes shifts and biexciton binding energies 


\begin{tabular}{|l|l|l|l|l|}
\hline \multicolumn{4}{|l|}{$\mathrm{CdSe}^{3}$} & $\mathrm{CdSe} / \mathrm{Cd}, \mathrm{Zn}, \mathrm{S}$ \\
\hline Radius & $1.5 \mathrm{~nm}$ & $2.1 \mathrm{~nm}$ & $2.8 \mathrm{~nm}$ & $2.4 \mathrm{~nm}$ \\
\hline$\delta_{\mathrm{x}}$ & $40 \mathrm{meV}$ & $25 \mathrm{meV}$ & $16 \mathrm{meV}$ & $30 \mathrm{meV}$ \\
\hline$\delta_{\mathrm{xx}}$ & $73 \mathrm{meV}$ & $57 \mathrm{meV}$ & $45 \mathrm{meV}$ & $57 \mathrm{meV}$ \\
\hline$\Delta^{\mathrm{E}}{ }_{\mathrm{xx}}$ & $50 \mathrm{meV}$ & $44 \mathrm{meV}$ & $37 \mathrm{meV}$ & $30 \mathrm{meV}$ \\
\hline$\Delta^{\mathrm{A}}{ }_{\mathrm{xx}}$ & $18 \mathrm{meV}$ & $12 \mathrm{meV}$ & $9 \mathrm{meV}$ & $3 \mathrm{meV}$ \\
\hline
\end{tabular}

Transient absorption spectroscopy can also be used to examine phonon modes in a nanocrystals sample, as seen for the $\mathrm{CdSe} / \mathrm{Cd}, \mathrm{Zn}, \mathrm{S}$ sample in figure $\mathrm{S} 3$. Here, a transition is pumped resonant with a peak in the absorption spectrum, and probe pulse is centered on the rising edge of the absorption and pump/probe time delay is scanned. The Fourier transform (FT) of the bleach recovery starting from the lowest point in the bleach is then taken. Amplitude of oscillations in the raw TA signal can give some insight as to the coupling strength, details given in a previous publication ${ }^{4}$; the residual of the FT to see peaks corresponding to specific phonon modes.

In figure S3a, it is evident that strong LO phonon peak at $208 \mathrm{~cm}^{-1}$ is present at all pump fluences. This same feature is also present when pumping the X2 and X3 transitions. This mode has not been observed when pumping the $\mathrm{X} 2$ and $\mathrm{X} 3$ transitions for CdSe core only NCs. When pumping at $400 \mathrm{~nm}$, this phonon is not observed. 

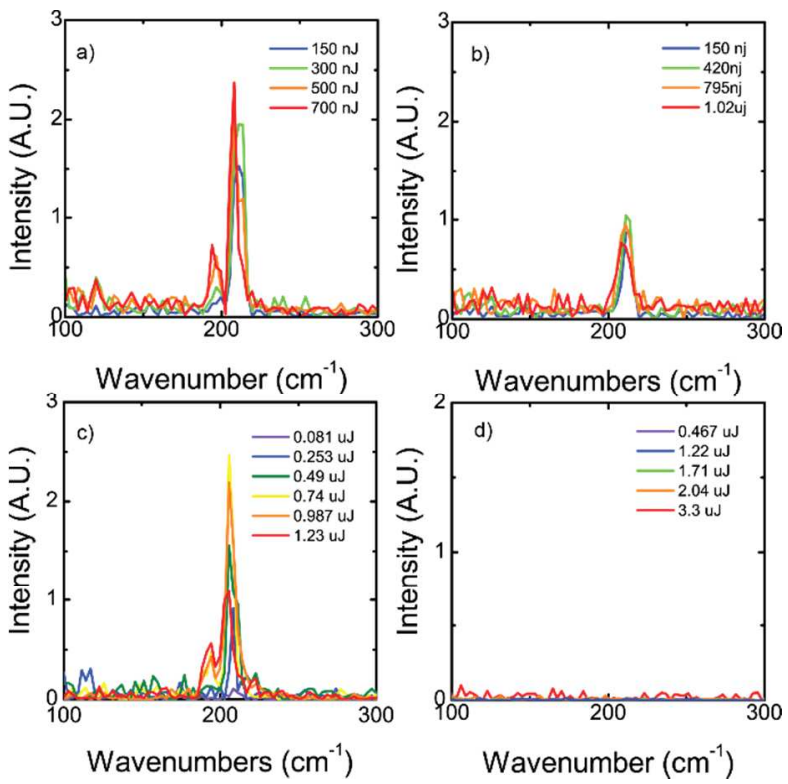

Figure S3. Fluence dependent phonons pumping at X1-X3 and continuum, panels a-d 

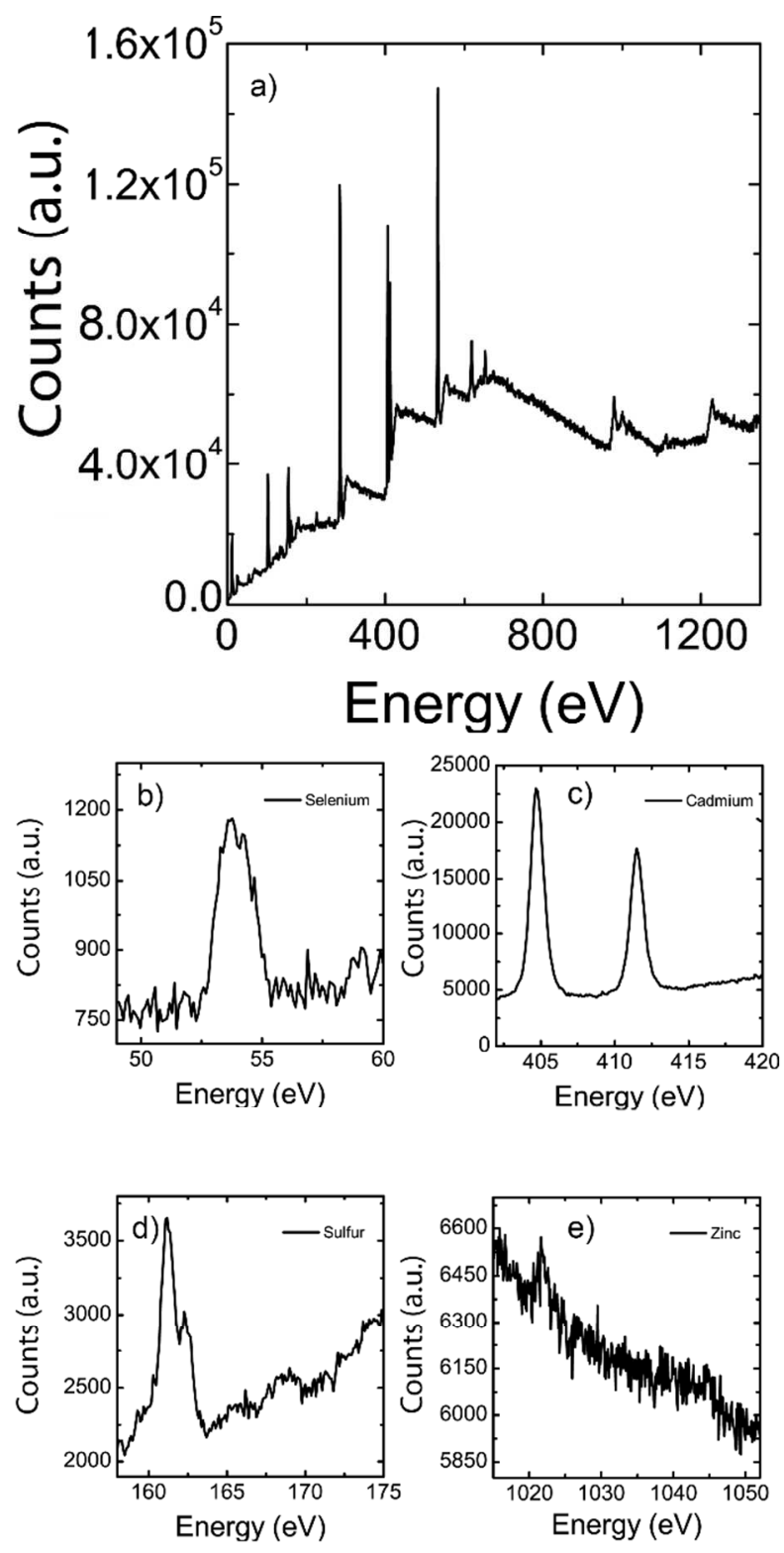

Figure S4a. Full XPS spectra of CdSe/Cd,Zn,S nanocrystal b. Selenium peak, c. Cadmium peaks, d. sulfur peak, e. zinc peaks

Figure S4 shows X-Ray photoelectron spectroscopy (XPS) spectra of the $\mathrm{CdSe} / \mathrm{Cd}, \mathrm{Zn}, \mathrm{S}$ sample. All characteristic peaks are seen in the broad spectra covering all relevant energies, figure S4a. Peaks for selenium, only present in the core, cadmium present in both the core and shell, as well as sulfur and small peaks corresponding to zinc, which are both only found in the 
shell are all evident in panels b-e. We see the presence of all component atoms, but it is difficult to make direct assumptions related to the composition of the core and the shell due to difficulty to calculate accurate penetration depths which is accentuated as these NCs are not perfectly spherical $^{5}$.

\section{References}

1. Klimov, V. I., Optical Nonlinearities and Ultrafast Carrier Dynamics in Semiconductor Nanocrystals. The Journal of Physical Chemistry B 2000, 104, 6112-6123.

2. Klimov, V. I.; McBranch, D. W.; Leatherdale, C. A.; Bawendi, M. G., Electron and Hole Relaxation Pathways in Semiconductor Quantum Dots. Physical Review B 1999, 60, 13740-13749.

3. Sewall, S. L.; Franceschetti, A.; Cooney, R. R.; Zunger, A.; Kambhampati, P., Direct Observation of the Structure of Band-Edge Biexcitons in Colloidal Semiconductor Cdse Quantum Dots. Physical Review $B$ 2009, 80, $0813101-4$.

4. Sagar, D. M.; Cooney, R. R.; Sewall, S. L.; Dias, E. A.; Barsan, M. M.; Butler, I. S.; Kambhampati, P., Size Dependent, State-Resolved Studies of Exciton-Phonon Couplings in Strongly Confined Semiconductor Quantum Dots. Physical Review B 2008, 77, 235321.

5. Walsh, B. R.; Saari, J. I.; Krause, M. M.; Nick, R.; Coe-Sullivan, S.; Kambhampati, P., Controlling the Surface of Semiconductor Nanocrystals for Efficient Light Emission from Single Excitons to Multiexcitons. The Journal of Physical Chemistry C 2015, 119, 16383-16389. 